\section{Regards sur l'économie allemande}

Bulletin économique du CIRAC

$89 \mid 2008$

Varia

\title{
Les PME allemandes veulent une baisse des prélèvements, pas des subventions
}

\section{Eberhard Vogt}

Traducteur : Isabelle Bourgeois

\section{OpenEdition \\ Journals}

Édition électronique

URL : http://journals.openedition.org/rea/2633

DOI : $10.4000 /$ rea. 2633

ISBN : 978-2-8218-0874-4

ISSN : 1965-0787

Éditeur

CIRAC

Édition imprimée

Date de publication : 1 décembre 2008

Pagination : 21-26

ISSN : 1156-8992

\section{Référence électronique}

Eberhard Vogt, "Les PME allemandes veulent une baisse des prélèvements, pas des subventions », Regards sur l'économie allemande [En ligne], 89 | décembre 2008, mis en ligne le 01 décembre 2010, consulté le 21 avril 2019. URL : http://journals.openedition.org/rea/2633 ; DOI : 10.4000/rea.2633 


\section{Les PME allemandes veulent une baisse des prélèvements, pas des subventions}

\section{Eberhard Vogt}

La crise commence à atteindre le Mittelstand (voir REA 83/07). Certes, pour l'instant, les carnets sont encore bien remplis, mais les entrées en commande tendent à se réduire, surtout dans le segment des équipementiers automobiles. Et même si les PME allemandes se voient de plus en plus souvent refuser leurs demandes de crédit bancaire - même par leur "banque-maison", leur établissement-partenaire de longue date -, elles n'en abordent pas moins la récession avec une relative confiance dans leur capacité à rebondir. C'est ce que révèle la dernière enquête sur les PME effectuée par la banque KfW, publiée le 28 novembre (KfW Mittelstandspanel 2008). Car «les PME allemandes ont su mettre à profit la période de la bonne tenue des activités pour fortement gagner en compétitivité : en développant une solide stratégie d'innovation, en améliorant leur ratio de capitaux propres et en s'orientant vers l'international. Elles sont donc bien armées pour résister à la crise financière ". C'est en ces termes que Wolfgang Kroh, membre du directoire de la KfW, résume les conclusions de l'enquête.

Si dans le cadre de son programme conjoncturel, le gouvernement fédéral vient de doter la KfW d'un fonds complémentaire destiné à soutenir les PME en suppléant à un crédit bancaire hésitant, il s'en tient là en ce qui concerne un 'plan d'urgence' spécifique. II est vrai aussi que les PME allemandes « ne réclament pas des subventions, auxquelles elles préfèrent la baisse du taux des prélèvements ", comme l'expliquait le 12 novembre Mario Ohoven, président de leur principale fédération, la Bundesverband mittelständische Wirtschaft (BVMW). Elles bénéficient en effet, depuis les années 1970, d'une politique diversifiée mais constante de soutien public (Mittelstandsförderung) qui se traduit entre autres par des mesures financières, et bien plus encore par le souci des gouvernements du Bund comme des Länder de ménager un environnement favorable à leur activité. Car outre-Rhin, le Mittelstand ne se résume pas seulement à la catégorie des entreprises petites et moyennes, où à l'un de ces éléments structurels sur lesquels repose la compétitivité allemande, il constitue aussi le modèle entrepreneurial par excellence et est, en ce sens, porteur de lien social.

Les PME constituant aussi le "potentiel économique de I'UE », comme le rappelle la fédération française CGPME, elles ont besoin d'un traitement particulier destiné à libérer leur potentiel de croissance. Les dispositions du «small business act » européen en cours d'adoption révèlent, malgré leur disparité, que ce qui bride le plus ce potentiel, c'est un environnement qui ne tient pas assez compte de leur situation spécifique: cette petite taille qui limite les économies d'échelle et qui confère au facteur temps une valeur stratégique particulière.

Nous avons demandé à $E$. Vogt, porte-parole de la fédération BVMW et rédacteur en chef du mensuel Der Mittelstand qu'elle édite (www.bvmw.de), d'évaluer la politique allemande en faveur des PME et de nous dresser le catalogue des principales revendications du Mittelstand à l'égard des pouvoirs publics. (IB)

L'économie allemande ne serait rien sans son Mittelstand, cette catégorie d'entreprises occupant jusqu'à 500 salariés et réalisant un chiffre d'affaires annuel 
Des conditions cadre privilégiant les grands groupes de moins de 50 millions $€$. En règle générale, ces $P M E$ sont statutairement indépendantes et dirigées par le propriétaire qui en est aussi le premier collaborateur. Ce patron-propriétaire porte seul le risque entrepreneurial, ce qui veut dire qu'il est non seulement responsable devant les tribunaux, mais aussi qu'il engage son patrimoine privé. En Allemagne, plus de trois millions d'entreprises répondent à cette définition, alors qu'on n'y recense que 4400 grands groupes. Autrement dit : 99,6\% des sociétés soumises à la taxe sur le chiffre d'affaires sont des PME. Elles occupent $70 \%$ de tous les actifs, forment $80 \%$ des apprentis, engendrent la moitié environ du PIB et réalisent quelque $50 \%$ des investissements bruts.

Pourtant, malgré leur rôle clef pour la compétitivité du site Allemagne, elles se heurtent à d'innombrables obstacles qui inhibent leur potentiel. En effet, les conditions cadre pour l'activité déterminées par les milieux politiques allemands ou l'action communautaire sont axées sur les besoins des grands groupes qu'elles privilégient de ce fait. Les PME cherchent donc à mieux faire valoir leurs intérêts auprès des milieux politiques grâce à l'action de leurs représentations professionnelles. Elles disposent de plusieurs lobbies, dont le plus important et le plus influent est la Fédération Bundesverband mittelständische Wirtschaft (BVMW). Créé en 1975, ce groupement d'intérêts multisectoriel et indépendant des partis politiques, compte aujourd'hui un peu plus de 150000 entreprises adhérentes ; près de 54000 d'entre elles sont membres de la BVMW, les autres étant affiliées dans ses douze organisations professionnelles partenaires. Le cœur de l'activité de notre fédération est la promotion d'une politique plus favorable au Mittelstand - en Allemagne comme dans l'Union européenne.

\section{Considérer la crise comme une opportunité}

Les PME commencent à ressentir les premiers effets d'un resserrement du crédit : soit leur «banque-maison » (Hausbank) rejette leur dossier, soit elle formule des conditions plus contraignantes. Lors d'une enquête que nous avons récemment effectuée auprès de nos membres, il est apparu qu'une entreprise sur deux se voit aujourd'hui confrontée à cette situation. Une sur cinq affirme avoir eu à ajourner ses projets d'investissement dans l'intérêt de la continuité des activités et de ses salariés. Enfin, $80 \%$ de nos membres s'attendent à des conditions encore plus restrictives au cours des mois à venir.

Une entreprise du Mittelstand s'adresse traditionnellement à un établissement des réseaux publics des Sparkassen ou des Volks- und Raiffeisenbanken [voir REA 88/08; IB]. Or les caisses d'épargne, contraintes de participer au sauvetage comme à la restructuration des banques publiques où elles sont parties prenantes, risquent de réduire en conséquence le volume de crédit accordé aux PME. Mais elles sont conscientes de leur rôle pivot et restent disponibles pour les PME. De leur côté, les entreprises ont commencé à multiplier au cours des dernières années leurs sources de financement, ce qui les rend moins tributaires du crédit bancaire classique.

Or toute crise, si elle est facteur de risque, présente aussi une opportunité. La meilleure politique consiste pour les PME à prendre conscience de leurs formidables atouts. Car la crise financière incite aussi les banques à redéfinir leurs activités, ce qui amène nombre d'entre elles à s'intéresser aux PME, ce qui ouvre à ces dernières de nouvelles perspectives de crédit - surtout si elles présentent un dossier porteur de valeurs immatérielles comme un portefeuille de marques, de brevets ou de licences. D'une manière plus générale, la crise forcera les PME à améliorer leur rating; elles y gagneront en efficience. Mais la principale chance que présente la crise est de révéler la nature plus structurelle des obstacles au développement du potentiel des PME, y compris aux yeux du monde politique: un état de la réglementation qui ne respecte pas suffisamment les besoins spécifiques des PME et un taux de prélèvements obligatoires désincitatif. La crise est donc aussi un appel à améliorer le cadre des activités. 


\section{Réduire le poids des prélèvements}

L'un de nos principaux objectifs est d'aboutir à une baisse du poids des prélèvements obligatoires qui représentent aujourd'hui $52,2 \%$ des coûts salariaux. Ce ratio place l'Allemagne dans le peloton de tête des pays de l'OCDE, loin devant la Suisse où la part des prélèvements est la plus faible en comparaison (29,6\% seulement).

Nous avons réalisé une enquête nationale auprès de nos adhérents pour recueillir leur réaction à un éventuel allégement des charges. Leurs réponses se passent de commentaire : les deux tiers (66\%) des PME disent qu'elles embaucheraient plus de collaborateurs si la charge fiscale baissait, et même la moitié d'entre elles si le niveau des cotisations se trouvait réduit.

Réduire le poids des prélèvements ne serait pas seulement bénéfique à l'emploi mais œuvrerait également pour une plus grande justice sociale. En effet, si le fisc allemand a pu accroître son encours de plus de 91 milliards $€$ entre 2004 et 2007, les ménages n'ont vu baisser dans le même temps leurs impôts que de 18 milliards $€$. II conviendrait en particulier de lutter contre un effet pervers du régime de l'impôt progressif et qui se traduit par le fait que, sous l'effet de l'inflation, toute hausse des revenus aboutit mécaniquement à une hausse larvée de l'impôt sur le revenu. En moyenne, une augmentation du salaire de $1 \%$ seulement accroît ainsi de 2 \% le montant des impôts prélevés.

La réduction de la charge fiscale pour les PME doit s'effectuer sous ses deux aspects : celui de l'impôt sur le revenu auquel sont soumises les entreprises en nom personnel, nombreuses dans le Mittelstand, et celui de l'impôt sur les sociétés. La réforme de ce dernier, telle qu'elle est intervenue au début de 2008, n'a en rien allégé la pression fiscale sur les PME ; pis encore, elle leur impose de surcroît le contre-financement de la réduction de l'impôt sur les bénéfices des sociétés de capitaux puisque la réforme supprime la dégressivité des amortissements. Le fait que celle-ci soit réintroduite pour deux ans dans le cadre du programme conjoncturel ne change pas la nature du problème. II convient donc de revoir certaines dispositions de la réforme de la fiscalité des entreprises. Par exemple, en ramenant à un niveau plus réaliste l'évaluation des baux, créditsbaux ou contrats de gérance intervenant dans le calcul de la taxe sur les bénéfices des professions industrielles et commerciales (Gewerbesteuer).

\footnotetext{
L'impôt sur les sociétés ne frappe que les sociétés de capitaux, minoritaires outre-Rhin. La majorité des entreprises du Mittelstand a en effet un statut de société de personnes. En droit fiscal, les patrons-propriétaires sont donc soumis à l'impôt sur le revenu en ce qui concerne le résultat de l'entreprise. La société en tant que telle est soumise à la taxe sur les bénéfices des professions industrielles et commerciales ; mais cette taxe est déductible du revenu imposable du propriétaire.

La récente réforme de la fiscalité des entreprises, dont le premier volet est entré en vigueur début 2008, le second s'appliquant à partir de 2009, s'est concentrée sur l'allègement de la charge fiscale pesant sur les sociétés de capitaux, éludant la question de fond qui réside dans la disparité des régimes fiscaux applicables aux sociétés et un système qui favorise actuellement les sociétés de capitaux (voir REA 84/07).

Après cette première phase de 'toilettage' est intervenue l'adoption d'un projet de réforme de l'imposition des successions, voté par le Bundestag le 27 novembre 2008 et approuvé par le Bundesrat le 5 décembre. Ce texte, qui entrera en vigueur le $1^{\text {er }}$ janvier 2009, apportera entre autres quelques allégements pour les successions d'entreprises.

Le code fiscal applicable aux PME n'en demeure pas moins d'une extrême complexité. L'une des raisons de cette complexité provient justement d'une articulation peu probante entre impôts sur le revenu et impôt sur les sociétés. Clarifier la situation est l'une des principales revendications formulées depuis longtemps par les milieux économiques, mais aussi les experts, à commencer par le Conseil des Sages qui y consacre régulièrement études et analyses, notamment dans les rapports annuels qu'il remet en fin d'année au gouvernement fédéral. (IB)
}

Plus fondamentalement, l'avenir des PME comme du sens de l'entreprise ou de la culture entrepreneuriale ne sera garanti en Allemagne qu'à la condition que la réforme en cours de la fiscalité des successions respecte mieux les contraintes comme les intérêts spécifiques des entreprises familiales. En l'état actuel, le projet de réforme condamne purement et simplement à la fermeture des dizaines de milliers de PME dans les années à venir si rien n'est entrepris pour aménager fiscalement le changement générationnel d'une manière à ce que l'héritier ne soit pas pénalisé par le fisc quand il reprend l'entreprise.

\section{La réforme de} l'impôt sur les successions pénalise les repreneurs 
Trop de rigidités

Le gouvernement fédéral a détaillé le coût de la paperasse...

... mais les mesures adoptées sont insuffisantes pour le réduire

Une réévaluation des coûts...
Le compromis que vient de conclure le gouvernement de coalition sur la réforme de l'impôt sur les successions apporte une réponse dans l'ensemble satisfaisante puisqu'il prend en considération les principales critiques formulées par le Mittelstand à l'encontre de la première version du projet de loi. Ainsi, il réduit de 15 à 10 ans la durée pendant laquelle l'héritier doit conserver le patrimoine de l'entreprise pour bénéficier d'un abattement fiscal. II eût mieux valu néanmoins que les législateurs renoncent purement et simplement à assortir de conditions la reprise d'une société par l'héritier.

\section{Assouplir la réglementation du travail}

C'est dans le domaine de la réglementation du travail que la propension de l'Etat à accroître les rigidités est la plus marquée. Nous nous contenterons de citer en exemple ici la législation en matière de protection contre le licenciement, particulièrement restrictive en comparaison européenne [voir Zumfelde, 2005 ; IB]. Ou de rappeler le projet d'instauration d'un salaire minimum légal auquel travaille actuellement le gouvernement fédéral [voir REA 85/07 et $86 / 08$; IB] et qui porte atteinte au principe constitutionnel de l'autonomie tarifaire (Tarifautonomie), c'est-à-dire à la responsabilité exclusive des fédérations patronales et des syndicats en matière de fixation des salaires. Ou encore les discussions en cours sur une réglementation plus contraignante de l'emploi intérimaire, au risque de brider le dynamisme d'un secteur en plein essor. Sans oublier la Loi générale sur l'égalité de traitement (Allgemeines Gesetz zur Gleichbehandlung, AGG) entrée en vigueur le 18 août 2006 et qui, au lieu de se contenter de transcrire les dispositions européennes en droit national a donné lieu à une surenchère en termes de non discrimination.

\section{Réduire les charges administratives}

Mais le principal frein à l'activité réside dans le coût des charges administratives. Les PME supportent en effet plus de 80 \% du coût global pour l'économie que génère l'activisme bureaucratique en Allemagne, soit 30 milliards $€$ au total selon l'estimation officielle publiée en avril 2008 par le gouvernement fédéral. L'office fédéral des Statistiques, de son côté, établit ces coûts à 40 , voire 50 milliards $€$.

Dans son rapport de 2007 sur la mise en oeuvre de la méthode des coûts standard (Standardkostenmodell), le gouvernement fédéral dresse une liste détaillée des dispositions et obligations légales comme des coûts qu'elles induisent pour les entreprises. A elle seule, par exemple, l'obligation de conserver les factures coûte annuellement 6,2 milliards $€$ aux entreprises. C'est l'obligation de loin la plus onéreuse. Elle est suivie en seconde et troisième position par la paperasse liée à la déclaration relative à la taxe sur le chiffre d'affaires (3,65 milliards $€)$ et à la production du bilan annuel (3,54 milliards $€)$. La disposition la moins coûteuse en comparaison ( 9 millions $€$ seulement) est celle qui fait obligation aux entreprises du secteur de la récupération du bois de tenir un registre comptable journalier (Betriebstagebuch).

Aucune des initiatives prises jusqu'ici pour réduire les charges administratives n'ayant été suivie d'effets notables, l'objectif que s'est fixé le gouvernement fédéral de réduire les coûts administratifs de $25 \%$ d'ici 2011 semble pour le moins ambitieux, si ce n'est même quelque peu contradictoire. Car en février 2007, le même gouvernement avait décidé, toujours dans l'objectif de ramener le poids de la paperasse à de plus justes proportions, "de procéder à une réévaluation du volume constaté des coûts administratifs afin d'identifier et de supprimer les coûts et charges inutiles ».

Mais au préalable avait été instituée une nouvelle bureaucratie : le Conseil national de contrôle des normes (Nationaler Normenkontrollrat), né en septembre 2006 en vertu de la Loi portant création de ce Conseil (Gesetz zur Errichtung 
eines Nationalen Normenkontrollrates, NKR) et composé de huit membres au mandat de cinq ans. Ce Conseil a pour mission de calculer les coûts administratifs induits par la législation en vigueur, mais aussi d'évaluer ceux qu'entraînerait la législation en projet. Jusqu'à présent, un bon tiers des quelque 11000 obligations légales d'information avaient été sous-évaluées quant à leur coût.

Dans le cadre de sa politique de réduction des obstacles administratifs, le gouvernement fédéral a pris diverses dispositions. D'une part, il a décidé des mesures d'accompagnement parmi lesquelles l'adoption en juillet 2008 d'un projet de Loi de simplification des procédures fiscales (Steuerbürokratieabbaugesetz, littéralement: Loi de réduction de la paperasse fiscale). D'autre part, il a fait adopter un train de trois lois visant à réduire les charges administratives pesant sur les PME (Mittelstandsentlastungsgesetze, MEG). Les deux premières (MEG I et MEG II) sont entrées en vigueur respectivement en août 2006 et en septembre 2007 (l'essentiel des dispositions de la MEG II), la troisième (MEG III), dont le texte a été adopté en conseil des ministres en juillet 2008, est actuellement en cours de lecture parlementaire. Elles suppriment un catalogue disparate de règlements isolés, d'obligations et de servitudes qui s'imposaient jusque là aux entreprises.

La loi MEG // a ainsi rendu caduques 17 dispositions, allégeant les charges administratives qui pesaient sur les PME. Désormais, par exemple, les petites entreprises n'ont plus à répondre que trois fois par an aux enquêtes statistiques ; quant aux créateurs d'entreprises, ils sont totalement exemptés de cette obligation d'information. Mais ces allégements, certes utiles, n'apportent qu'une faible réduction des coûts, d'un volume de 60 millions $€$ seulement.

La loi MEG III, en cours d'adoption, n'apporte pas, elle non plus, d'amélioration sensible. Certes, elle supprimera pour 460000 entreprises artisanales l'obligation d'information dans le cadre du recensement sectoriel qu'effectue Destatis, mais elle ne se traduit que par un allègement de 24 millions $€$. Certes, 175000 propriétaires d'agences de voyage, de teintureries et d'ateliers de réparation seront dispensés d'apposer sur leur pas de porte une plaque portant mention de leur nom intégral, ce qui réduit les coûts de 66 millions $€$. Mais le catalogue des dispositions disparates ainsi abrogées ne réduira les coûts administratifs que de 100 millions $€$ au total.

Au vu de cet état de fait, on peut se demander si le train de mesures des trois lois MEG permettra d'atteindre son ambitieux objectif affiché, à savoir réduire de 850 millions $€$ le coût des charges administratives pour les PME. D'autant plus que, depuis décembre 2006, la charge administrative s'est en réalité accrue : certes, 130 obligations légales d'information ont été supprimées depuis, et 230 autres modifiées, mais dans le même temps, 500 nouvelles ont été adoptées. Jusqu'à mi-mandat, le gouvernement de coalition de Berlin avait fait voter quelque 700 lois ; les trois quarts d'entre elles ont engendré des coûts administratifs supplémentaires.

\section{Investir plus et mieux dans la formation}

Le Mittelstand manque cruellement de main d'œuvre qualifiée : 400000 emplois sont vacants. Cette situation inquiétante pour la compétitivité de notre économie a pour principale origine un niveau de dépenses d'éducation et de formation trop faible en comparaison internationale [voir à ce sujet REA 88/08; $I B]$. L'Allemagne n'investit que $5 \%$ de son PIB dans l'éducation et la formation, alors que les Etats-Unis ou la Corée du Sud y consacrent plus de $7 \%$.

Or en même temps, la plupart des écoles comme des universités dispensent des qualifications qui ne tiennent pas compte des besoins de l'économie. C'est ainsi que, au total, $8 \%$ des élèves quittent le système scolaire sans diplôme, que $17 \%$ des jeunes sortent du système de formation professionnelle sans métier [voir REA 75/06 ; IB] et que $30 \%$ des étudiants abandonnent leurs études. La souveraineté des Länder dans le domaine scolaire et universitaire (le Bund
... a débouché sur 3 lois d'allègement spécifiques aux PME

Elles n'apportent pas d'amélioration sensible

Redoubler d'efforts pour former une main d'œuvre qualifiée 
La réforme de l'assurance maladie renchérit le coût du travail n'est pas compétent) constitue elle aussi un handicap pour la compétitivité du système allemand de formation, puisqu'elle multiplie par seize les approches et les budgets en matière de politique éducative.

\section{Réduire les charges sociales (assurance maladie)}

La principale mesure de la réforme de l'assurance maladie qui entrera en vigueur le 1er janvier 2009 est l'institution d'un Fonds santé d'une haute complexité [voir dans ce numéro; IB]. Pour la première fois dans I'histoire de la RFA, le gouvernement fédéral a fixé un taux de cotisation unique pour toutes les caisses d'assurance maladie du régime légal. II est supérieur de 0,6 point de pourcentage à l'actuel taux moyen qui se situe à $14,8 \%$ du salaire brut.

Or, dans la mesure où les employeurs supportent la moitié des cotisations prélevées sur les salaires de leurs salariés, ils verront s'accroître arithmétiquement leurs coûts salariaux annexes, ce qui renchérira le coût du travail en Allemagne. Et parmi les PME, ce sont surtout les entreprises artisanales qui verront ainsi s'alourdir considérablement leurs charges. Ces conséquences indirectes de la réforme ne seront pas sans effet sur la compétitivité globale du site Allemagne.

LES CHANTIERS DE REFORMES A ENGAGER sont innombrables et urgents. Mais avant tout, il convient que le monde politique change de perspective et développe une nouvelle approche de son action pour que l'Allemagne reste compétitive dans le jeu de la concurrence européenne et mondiale. Et il convient de ménager un plus grand espace de liberté au Mittelstand afin que les PME puissent déployer et faire fructifier pleinement leur potentiel d'innovation.

Faut-il rappeler que l'évolution au jour le jour des cotations boursières n'est pas une préoccupation majeure pour les petites et moyennes entreprises ? Leur stratégie est orientée sur le long terme, sur la patiente construction d'une activité stable et pérenne, donc sur son développement durable. En tant qu'acteur économique, le Mittelstand assume son rôle de partenaire des pouvoirs publics. Mais de ce fait même, il attend du politique qu'il assume lui aussi son rôle, qui est de créer un environnement réglementaire favorable à l'activité, en particulier à celle du Mittelstand. De plus, dans la situation de crise que nous traversons actuellement, une telle politique se révélera plus efficace que tout plan de soutien sectoriel ou de subventions aux entreprises.

Traduction : I. Bourgeois

\section{Indications bibliographiques}

BOURGEOIS I., LASSERRE R., "Les PME allemandes : acteurs de la mondialisation », Regards sur l'économie allemande, $n^{\circ} 83 / 07$

"Bundeskabinett beschließt Drittes Mittelstandsentlastungsgesetz », communiqué en date du 23-07-2008 publié par le ministère fédéral de l'Economie (www.bmwi.de) et enrichi de liens vers les textes de ce projet de loi ainsi que des deux lois précédentes

Bürokratieabbau. Jetzt Entscheidungen treffen. Jahresbericht 2008 des Nationalen Kontrollrats, juin 2008 (www.normenkontrollrat.bund.de)

Bürokratiekosten : Erkennen-Messen-Abbauen. Bericht der Bundesregierung 2007 zur Anwendung des Standardkosten-Modells, www.bundesregierung.de

CGPME, Simplifier la vie des PME - 10 mesures, et Pour un Small Business Act à la française, documents présentant les positions de la CGPME, publiés respectivement en août et juin 2007 (www.cgpme.fr)

DEUTSCHE BUNDESBANK, "Zur wirtschaftlichen Situation kleiner und mittlerer Unternehmen in Deutschland seit 1997 », Monatsbericht, décembre 2006

KFW, Mittelstandspanel 2008, novembre 2008 (www.kfw.de)

PME 2008. Rapport OSEO sur l'évolution des PME, Paris, 2008

ZUMFELDE M., "L'assouplissement du droit allemand des licenciements ", in BOURGEOIS I. (dir.), Le modèle social allemand en mutation, Travaux et Documents du CIRAC, 2005

Zwischenbericht des Staatssekretärsausschusses Bürokratieabbau an das Bundeskabinett. Sachstand über das Regierungsprogramm „Bürokratieabbau und bessere Rechtsetzung“, avril 2008 (www.bundesregierung.de/buerokratieabbau) 